\title{
Die Zürcher Wälder um 1823 - Betriebsformen und Baumarten
}

Mirjam Bader

Urs Gimmi

Matthias Bürgi
Eidgenössische Forschungsanstalt für Wald, Schnee und Landschaft $(\mathrm{CH})$

Eidgenössische Forschungsanstalt für Wald, Schnee und Landschaft $(\mathrm{CH})$

Eidgenössische Forschungsanstalt für Wald, Schnee und Landschaft $(\mathrm{CH})^{*}$

\section{Die Zürcher Wälder um 1823 - Betriebsformen und Baumarten}

Aus den Visitationsberichten von 1823 sollen Bewirtschaftung und Baumartenzusammensetzung der öffentlichen Wälder im Kanton Zürich im frühen 19. Jahrhundert rekonstruiert werden. Dazu werden die Verteilung der Betriebsformen im ganzen Kanton und die Baumartenzusammensetzung in 31 ausgewählten Gemeinden im Zürcher Unterland analysiert. Ausserdem wird für diese Gemeinden ein Vergleich mit der heutigen Baumartenzusammensetzung durchgeführt. Die Auswertungen zeigen, dass der Mittelwald im frühen 19. Jahrhundert die wichtigste Betriebsform im Kanton Zürich war. Die Betriebsformen unterscheiden sich sehr stark in ihrer Baumartenzusammensetzung, wobei die grösste Vielfalt an Baumarten in Nieder- und Mittelwäldern zu finden ist. Die Resultate legen die Vermutung nahe, dass kein klarer Zusammenhang zwischen der Baumartenzusammensetzung und natürlichen Faktoren wie Klima, Topografie und Boden besteht. Daraus kann geschlossen werden, dass die Bewirtschaftung bereits im frühen 19. Jahrhundert einen grösseren Einfluss auf die Baumartenzusammensetzung hatte als die naturräumlichen Voraussetzungen. Die Baumartenzusammensetzung im Zürcher Unterland hat sich in den letzten 200 Jahren stark verändert. Vermutlich sind die Wälder heute naturnaher als 1823, was allerdings nicht mit einer grösseren Artenvielfalt gleichgesetzt werden kann. Das Wissen über die frühere Baumartenzusammensetzung kann daher von praktischem Nutzen bei der Revitalisierung ehemaliger Niederund Mittelwälder sein, welche wertvolle Habitate für seltene Tier- und Pflanzenarten bieten.

Keywords: forest history, historical forest ecology, forest management plans, conservation, canton Zurich, Switzerland

doi: $10.3188 /$ szf.2015.0024

*Zürcherstrasse 111, CH-8903 Birmensdorf, E-Mail matthias.buergi@wsl.ch

$\mathrm{D}$ ie meisten Waldökosysteme sind stark durch vielfältige menschliche Nutzungen geprägt (Noble \& Dirzo 1997). Da sich solche Ökosysteme meist langsam und über lange Zeiträume hinweg entwickeln, sind menschliche Einflüsse auch nach vielen Jahren noch sichtbar. Wälder können daher ein Spiegel vergangener kultureller, ökonomischer und gesellschaftlicher Entwicklungen sein. Aufgrund historischen Wissens werden auch Szenarien für das zukünftige Aussehen des Waldes erarbeitet (Zingg 2001). Aufgrund der Untersuchung der Reaktion von Waldökosystemen auf menschliche Eingriffe in der Vergangenheit wird beispielsweise versucht, geeignete Strategien für den Umgang mit dem Klimawandel zu finden (Sjölund \& Jump 2013). Ausserdem kann die Kenntnis der vergangenen Waldentwicklung einen wichtigen Beitrag zum Artenschutz leisten, da die Artenvielfalt mit der Waldstruktur zusammenhängt (Schütz 2005).

Mit der Rekonstruktion der früheren Baumartenzusammensetzung befassen sich zahlreiche Arbeiten aus den USA und Europa. In den USA liegt mit den Vermessungsaufnahmen eine besondere Art von Quellen zur historischen Baumartenzusammensetzung vor. Diese erfolgten vor der europäischen Besiedelung und sind daher oft schon für die zweite Hälfte des 18. Jahrhunderts verfügbar. In diesen Aufnahmen wurden die Grenzen der einzelnen Abteilungen durch sogenannte «witness trees» markiert (Cogbill et al 2002, Bürgi et al 2000). Verschiedene Arbeiten versuchen, daraus die Baumartenzusammensetzung vor der grossflächigen Besiedelung durch europäische Auswanderer zu rekonstruieren (z.B. Vadeboncoeur et al 2012, Sholes \& Hawkins 2007). Axelsson \& Östlund (2001) befassen sich mit der Veränderung von Baumartenzusammensetzung und Landschaftsmustern in Schweden, indem sie Katasterpläne und frühe Forstinventare aus dem 19. Jahrhundert mit modernen Aufnahmen vergleichen. Rüther (2010) beschreibt die Baumartenzusammensetzung des Leopolds-Hochwaldes in Bayern vom 15. bis ins 19. Jahrhundert, allerdings ohne die Baumartenanteile zu quantifizieren. Neben Waldbeschreibungen wertete er auch Gerichtsakten und Grundbücher aus. 


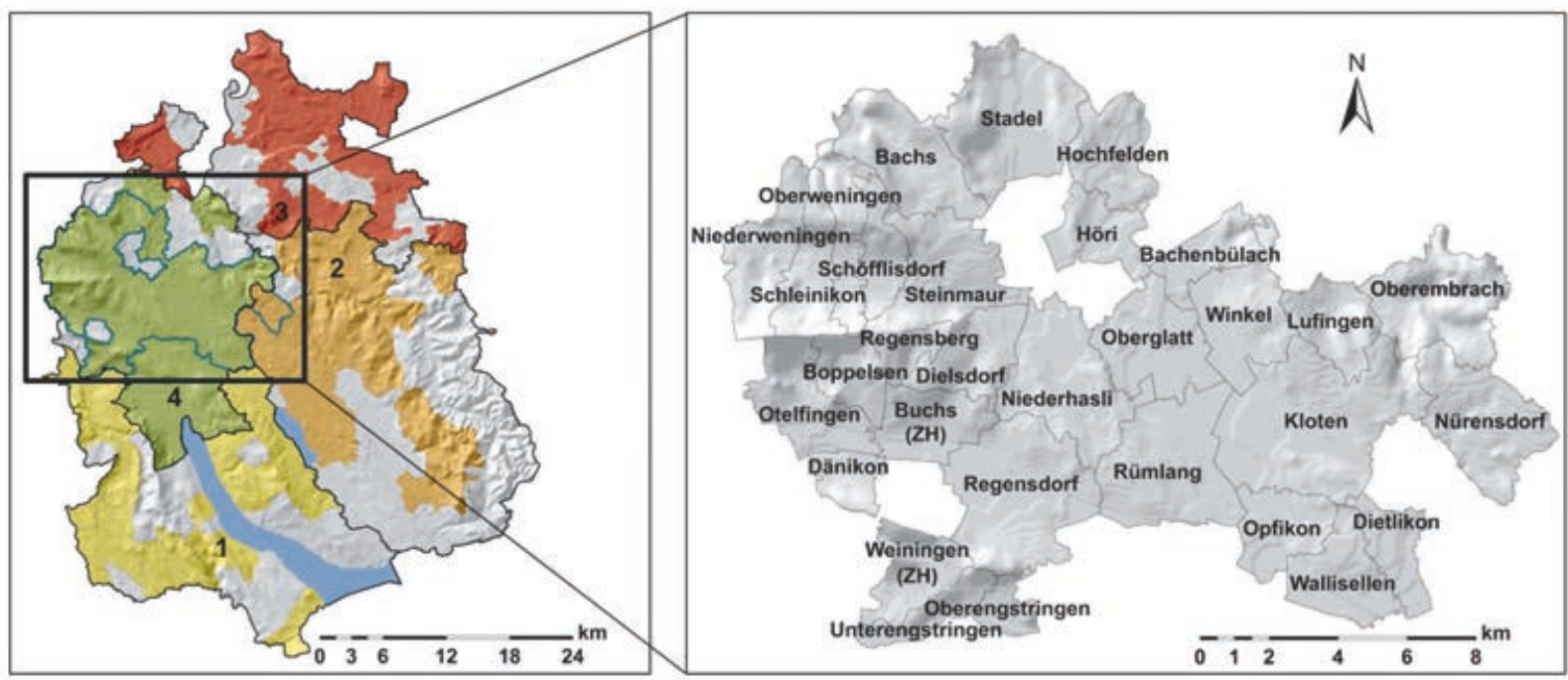

Abb 1 Untersuchte Gemeinden im Kanton Zürich mit mindestens einem verfügbaren Visitationsbericht und ihre Einteilung in die vier damaligen Forstkreise (links); Gemeinden im Zürcher Unterland mit genauen Angaben zur Baumartenzusammensetzung (rechts). Kartengrundlage: BFS GEOSTAT/Bundesamt für Landestopografie, Kreisgrenzen nach Weisz et al (1983).

Bürgi (1998) rekonstruierte aus verschiedenen Waldwirtschaftsplänen die Veränderung der Baumartenzusammensetzung im Kanton Zürich. Dabei konzentrierte er sich auf das 20. Jahrhundert. Ähnliche Untersuchungen über die Baumartenverteilung Anfang des 19. Jahrhunderts liegen bisher nicht vor.

Für den Kanton Zürich finden wir uns in der vorteilhaften Lage, dass bereits 1823 die meisten öffentlichen Wälder in Vorläufern der Waldwirtschaftspläne, den sogenannten Visitationsberichten, erfasst und beschrieben worden sind. Im vorliegenden Artikel rekonstruieren wir basierend auf diesen einzigartigen Quellen die damaligen Betriebsformen und die Baumartenzusammensetzung der öffentlichen Wälder im Kanton Zürich respektive im Zürcher Unterland.

\section{Material und Methoden}

\section{Untersuchungsgebiet}

Für 108 (63.5\%) der insgesamt 170 Gemeinden im Kanton Zürich sind Visitationsberichte verfüg-

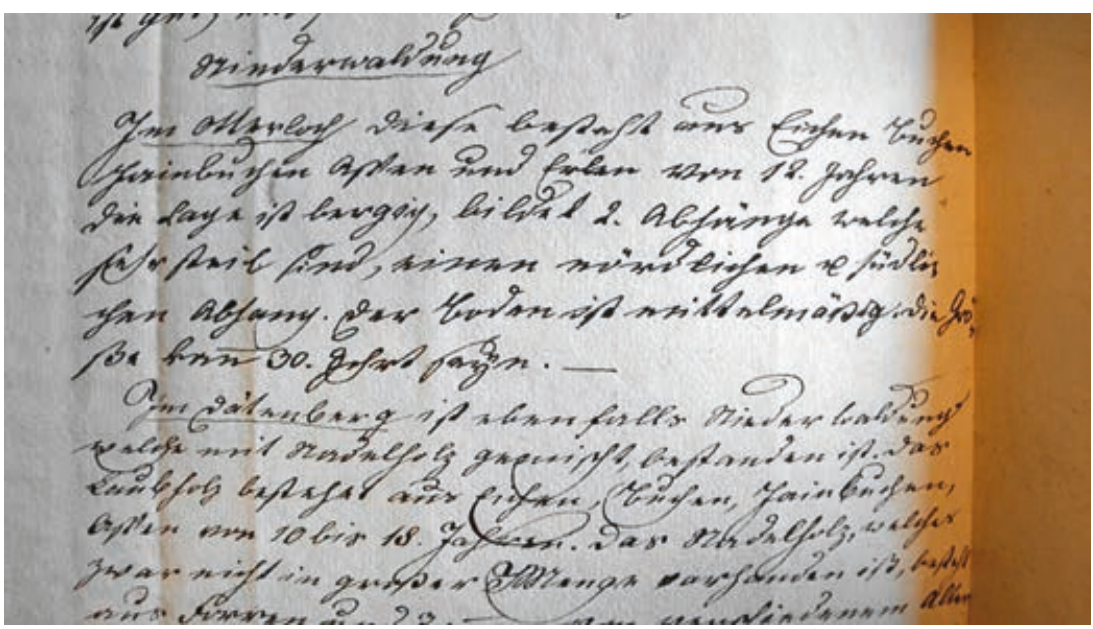

Abb 2 Ausschnitt aus dem Visitationsbericht der Gemeinde Oberstammheim, 1823. bar, wodurch das Untersuchungsgebiet definiert ist (Abbildung 1). Für jede untersuchte Gemeinde liegt mindestens ein Visitationsbericht vor, wobei die öffentlichen Wälder grösserer Gemeinden meist in mehreren Visitationsberichten beschrieben werden. Die betrachteten Gemeinden liegen mehrheitlich im Norden und Westen des Kantons, wo der Privatwaldanteil am geringsten ist. Für die Gemeinden im Zürcher Oberland mit ihren hohen Privatwaldanteilen sind hingegen meist keine Visitationsberichte verfügbar. Die meisten Auswertungen zur Baumartenzusammensetzung wurden für 31 ausgewählte Gemeinden im Zürcher Unterland durchgeführt. Für diese Gemeinden liegen besonders genaue Beschreibungen der Baumartenzusammensetzung vor (Abbildung 1).

\section{Quellen}

Hauptquellen der vorliegenden Arbeit sind die Visitationsberichte von 1823. ${ }^{1}$ Dabei handelt es sich um frühe Waldwirtschaftspläne, in welchen erstmals alle öffentlichen Wälder des Kantons Zürich beschrieben werden. Nachdem die Kantone durch die Mediationsakte von 1803 wieder mehr Selbstständigkeit erhalten hatten, wurde im Kanton Zürich nach und nach eine neue Forstorganisation geschaffen. Durch verschiedene Vorschriften sollten unter anderem die Nebennutzungen in den Wäldern eingeschränkt werden. Dazu wurden 1822 vier Forstkreise geschaffen, welche von je einem Forstmeister beaufsichtigt wurden. Um einen Überblick über die Wälder zu erhalten, verfassten die Forstmeister Rudolf Steiner, Jacob Hertenstein, Ulrich Meister und Kaspar Obrist im Jahr 1823 die Visitationsberichte. Ihre praktische Ausbildung hatten die Forstmeister im Thüringer Wald absolviert, da zu dieser Zeit noch keine Forstschulen in der Schweiz existierten (Weisz

1 Visitationsberichte: StAZH Z 31, diverse Signaturen 


\begin{tabular}{|l|c|c|c|c|c|c|c|c|}
\hline \# BA & $\mathbf{1}$ & $\mathbf{2}$ & $\mathbf{3}$ & $\mathbf{4}$ & $\mathbf{5}$ & $\mathbf{6}$ & $\mathbf{7}$ & $\mathbf{8}$ \\
\hline 1 & $100 \%$ & & & & & & & \\
\hline 2 & $60 \%$ & $40 \%$ & & & & & & \\
\hline 3 & $50 \%$ & $30 \%$ & $20 \%$ & & & & & \\
\hline 4 & $40 \%$ & $30 \%$ & $20 \%$ & $10 \%$ & & & & \\
\hline 5 & $30 \%$ & $20 \%$ & $20 \%$ & $20 \%$ & $10 \%$ & & & \\
\hline 6 & $30 \%$ & $20 \%$ & $20 \%$ & $10 \%$ & $10 \%$ & $10 \%$ & & \\
\hline 7 & $20 \%$ & $20 \%$ & $20 \%$ & $10 \%$ & $10 \%$ & $10 \%$ & $10 \%$ & \\
\hline 8 & $20 \%$ & $20 \%$ & $10 \%$ & $10 \%$ & $10 \%$ & $10 \%$ & $10 \%$ & $10 \%$ \\
\hline
\end{tabular}

Tab 1 Gewichtungsschema für die Quantifizierung der Baumartenanteile. Lesehilfe: 1. Spalte: Anzahl genannte Baumarten, 1. Zeile: Stelle der Nennung.

et al 1983). Die Visitationsberichte wurden von Hand geschrieben (Abbildung 2) und können im Staatsarchiv Zürich eingesehen werden. Der Aufbau der Visitationsberichte basiert auf einer Instruktion des Oberforstamtes. ${ }^{2}$ Demnach sollen die Berichte Angaben zur Grösse der Waldungen machen sowie die Betriebsformen, das Alter und die Baumarten in den verschiedenen Abteilungen beschreiben. Danach folgen eine Beschreibung der bisherigen Bewirtschaftung sowie Vorschläge zur zukünftigen Behandlung des Waldes. Quantitative Angaben zum Vorrat und zu den Baumartenanteilen fehlen in den Visitationsberichten weitgehend. Auch ist die Beschreibung der Baumarten abhängig vom Verfasser unterschiedlich ausführlich und präzis.

Um die damalige Baumartenzusammensetzung mit der heutigen vergleichen zu können, wurden zusätzlich Daten aus der Regionalwaldinventur des Kantons Zürich der Jahre 2005 bis 2013 ausgewertet. ${ }^{3}$ In den Vergleich einbezogen wurden in der vorliegenden Arbeit nur Daten aus dem öffentlichen Wald, unter der Annahme, dass sich die Besitzverhältnisse nicht wesentlich verändert haben.

\section{Vorgehen}

Die handschriftlichen Visitationsberichte wurden im Staatsarchiv Zürich transkribiert. Insgesamt wurden 151 Visitationsberichte aus 108 Gemeinden erfasst und ausgewertet. Da sie meist in Form von Fliesstext vorliegen, mussten sie für die Auswertung in eine tabellarische Form gebracht werden. Dazu wurde jede erwähnte Abteilung mit ihrem Flurnamen und ihrer Flächengrösse einer der drei Betriebsformen Niederwald, Mittelwald und Hochwald zugeordnet. Die Betriebsform Hochwald wurde weiter in drei Unterkategorien eingeteilt: reine Nadelhochwälder, reine Laubhochwälder und gemischte Hochwälder. Basierend auf den Flächenangaben konnten die Anteile der Betriebsformen berechnet werden. Allerdings sind die Betriebsformen von den damaligen Forstmeistern nicht einheitlich bezeichnet worden. So ergab sich bei der Auswertung oft das Problem, dass als Niederwald bezeichnete Bestände dennoch Angaben zum Oberholz enthielten. In dieser Arbeit werden solche Bestände konsequent als
Mittelwälder klassiert, obwohl sie wahrscheinlich oft sehr wenig Oberholz enthielten und somit eher den Charakter eines Niederwaldes hatten. Allerdings konnten nur so alle Angaben zu den Baumarten erfasst werden. Als Niederwälder bezeichnet werden hier also nur Bestände, die vollständig oberholzfrei sind (vgl. Bürgi 1998).

In einem nächsten Schritt mussten die Angaben zu den Baumarten quantifiziert werden. Dabei wurde von der Annahme ausgegangen, dass die in einer Aufzählung zuerst genannten Baumarten häufiger sind als die zuletzt genannten (vgl. Dupuis et al 2011, Gimmi et al 2010). Basierend auf dieser Annahme wurde ein Gewichtungsschema (Tabelle 1) entworfen, welches in allen Auswertungen zur Anwendung kam. Die Baumartenanteile pro Abteilung wurden anschliessend flächengewichtet und für jede Gemeinde zusammengefasst. Im Mittelwald wurden die Baumartenzusammensetzung des Ober- und jene des Unterholzes zunächst einzeln berechnet und in einem zweiten Schritt zusammengefasst. Dabei wurden die beiden Schichten gleich gewichtet. Da der Vorrat im Oberholz sehr unterschiedlich gewesen sein muss (Bürgi 1998) und die Visitationsberichte keine genaueren Angaben dazu enthalten, erscheint diese Vorgehensweise am zweckmässigsten. Für die Auswertung wurden nur jene Baumarten berücksichtigt, die in mindestens zehn Abteilungen mit einem Anteil von mehr als 10\% vorkamen. Laub- und Nadelbaumarten mit kleineren Anteilen wurden unter den Oberbegriffen «Laubholz allgemein» respektive «Nadelholz allgemein» zusammengefasst. Diesen Kategorien zugewiesen wurden auch alle nicht eindeutigen Angaben der Visitationsberichte. Bei den Laubbäumen war dies oft der Fall bei frischen Schlägen, wo die Baumarten nicht unterschieden worden waren. Nadelbäume waren häufig als «Tannholz» bezeichnet, was auch keine Unterscheidung der Arten erlaubte.

Für die Untersuchung der Veränderung der Baumartenzusammensetzung wurden die auf Aufzählungen basierenden relativen Häufigkeiten der Baumarten des Jahres 1823 für 31 ausgewählte Gemeinden im Zürcher Unterland mittels WilcoxonTest mit Grundflächenanteilen aus der aktuellen Regionalwaldinventur verglichen (vgl. Vorgehen von Terrail et al 2014). Für die grafische Darstellung der Baumartenzusammensetzung und der Betriebsformen auf verschiedenen Karten kam die Software ArcGIS 10.2 (ESRI 2013) zum Einsatz. Statistische Auswertungen erfolgten unter Verwendung von R 2.15.1 (The R Foundation for Statistical Computing 2012).

\footnotetext{
2 Circulare an die Herren Forstmeister, 1823 (Allgemeiner Auftrag zur Untersuchung der Gemeinds Waldungen): StAZH OO 67.1, Befehlbuch über die Gemeindewaldungen, Band I (1823-1930), S. 3f.

3 Amt für Landschaft und Natur, Abteilung Wald
} 


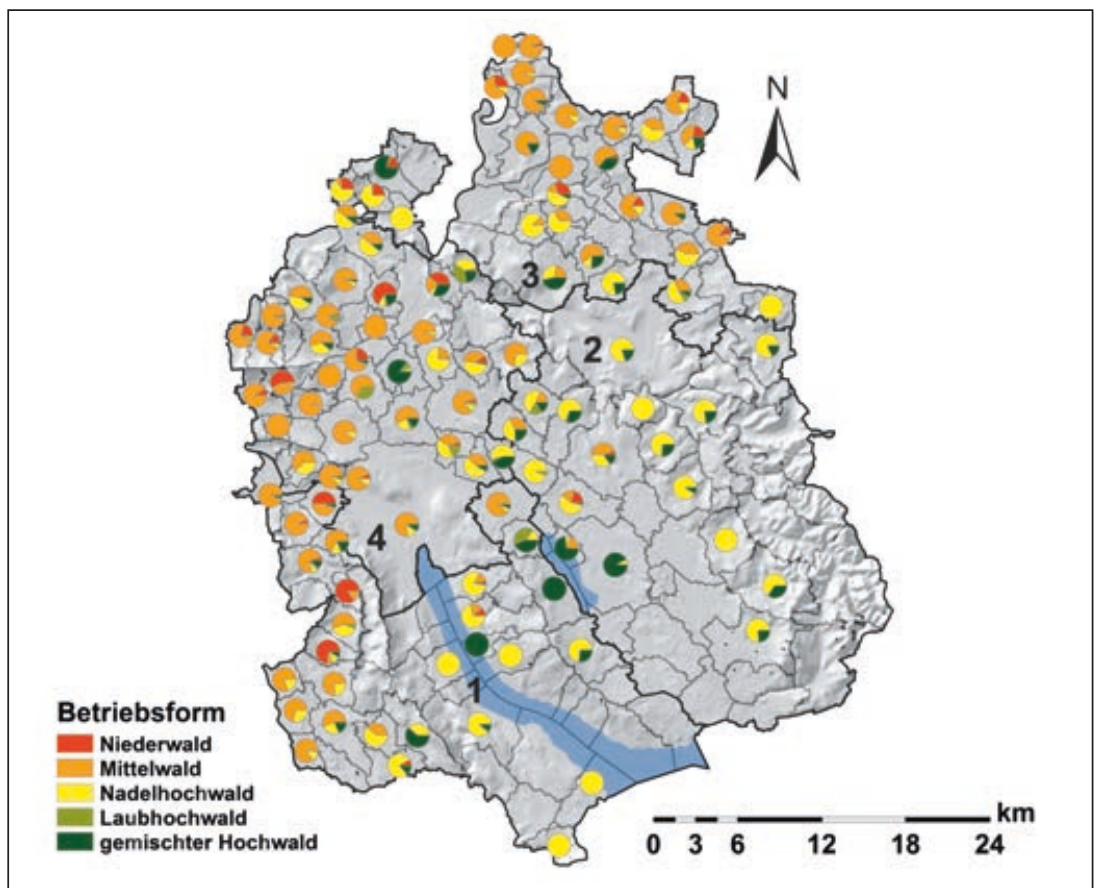

Abb 3 Verteilung der Betriebsformen im Jahr 1823 in den untersuchten Gemeinden. Kartengrundlage: BFS GEOSTAT/Bundesamt für Landestopografie, Kreisgrenzen nach Weisz et al (1983).

\section{Resultate}

\section{Betriebsformen im Kanton Zürich}

Einen Überblick über die im Jahr 1823 in den 108 untersuchten Zürcher Gemeinden verwendeten Betriebsformen zeigt Abbildung 3. Niederwälder ma-
Abb 4 Flächenanteile und Verteilung der wichtigsten Baumarten im Zürcher Unterland im Jahr 1823. Kartengrundlage: BFS GEOSTAT/Bundesamt für Landestopografie. chen einen geringen Anteil der Waldfläche aus, wobei nur einige wenige Gemeinden einen Niederwaldanteil von mehr als 50\% aufweisen. Ihre Verteilung folgt allerdings keinem klar erkennbaren räumlichen Muster.

Mittelwälder waren vor allem im Norden und im Westen des Kantons verbreitet. Der grösste Anteil an Mittelwäldern findet sich im damaligen Forstkreis 4. In den etwas höher gelegenen Gebieten Richtung Zürcher Oberland (Forstkreis 2) sind die meisten Bestände reine Nadelhochwälder. Solche kommen hingegen im Forstkreis 4 eher selten vor. Reine Laubhochwälder spielen in allen damaligen Forstkreisen nur eine untergeordnete Rolle mit einem Flächenanteil von höchstens 3\% im Forstkreis 4. Gemischte Hochwälder finden sich praktisch nur im zentralen Teil des Kantons. Dort nehmen sie aber teilweise einen grossen Anteil an der Waldfläche ein. Über den ganzen Kanton gesehen ist der Mittelwald mit einem Anteil von 48\% die häufigste Betriebsform, gefolgt vom Nadelhochwald mit einem Anteil von 31\%.

\section{Baumartenzusammensetzung im Zürcher Unterland}

Die weitaus häufigste Baumart im Zürcher Unterland mit einem Anteil von 32\% an der Gesamtfläche ist die Eiche (Abbildung 4). Die Föhre und die Fichte sind mit Anteilen von 12\% respektive 13\% vertreten. Während die Föhrenvorkommen eine eher
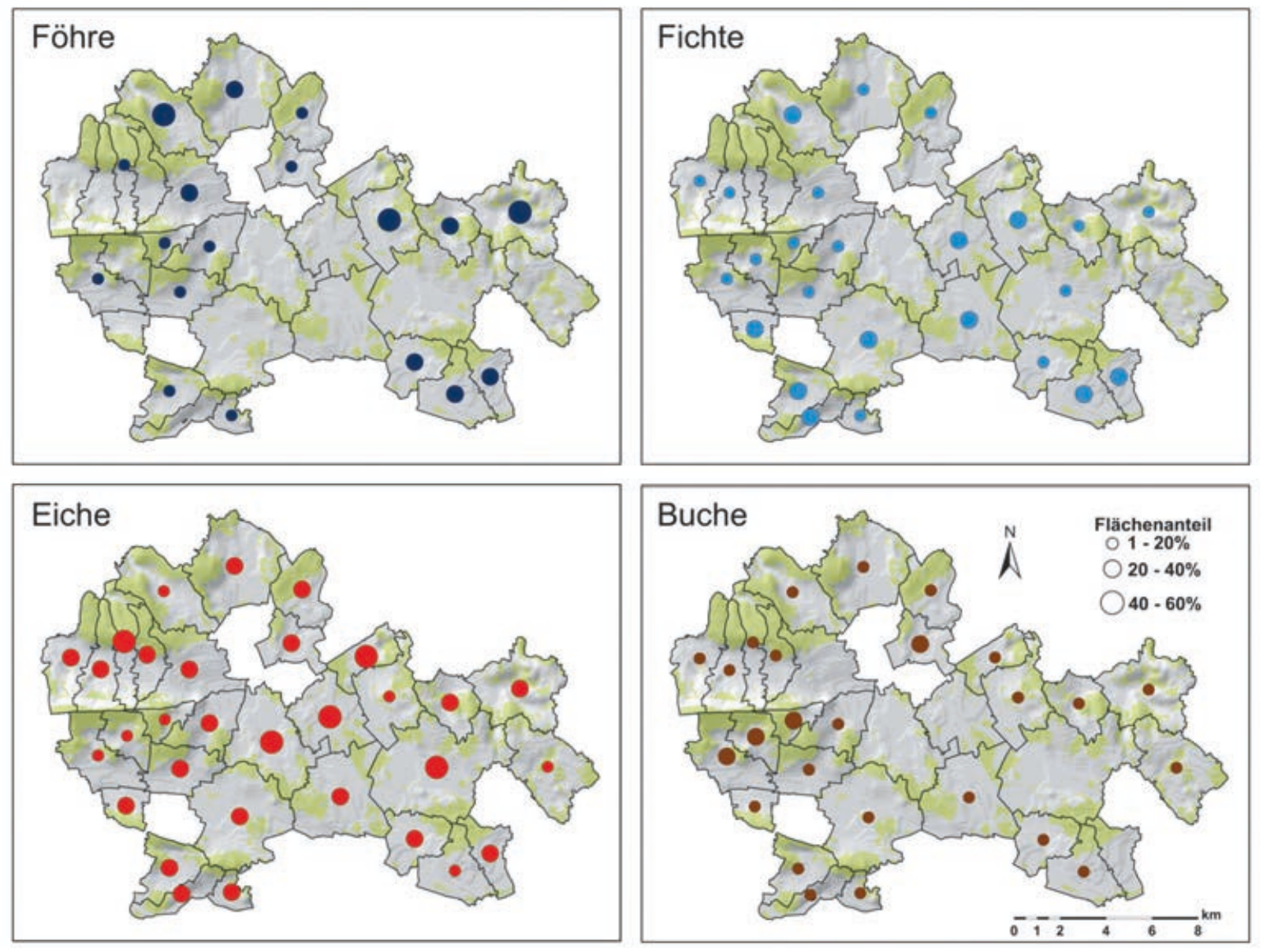


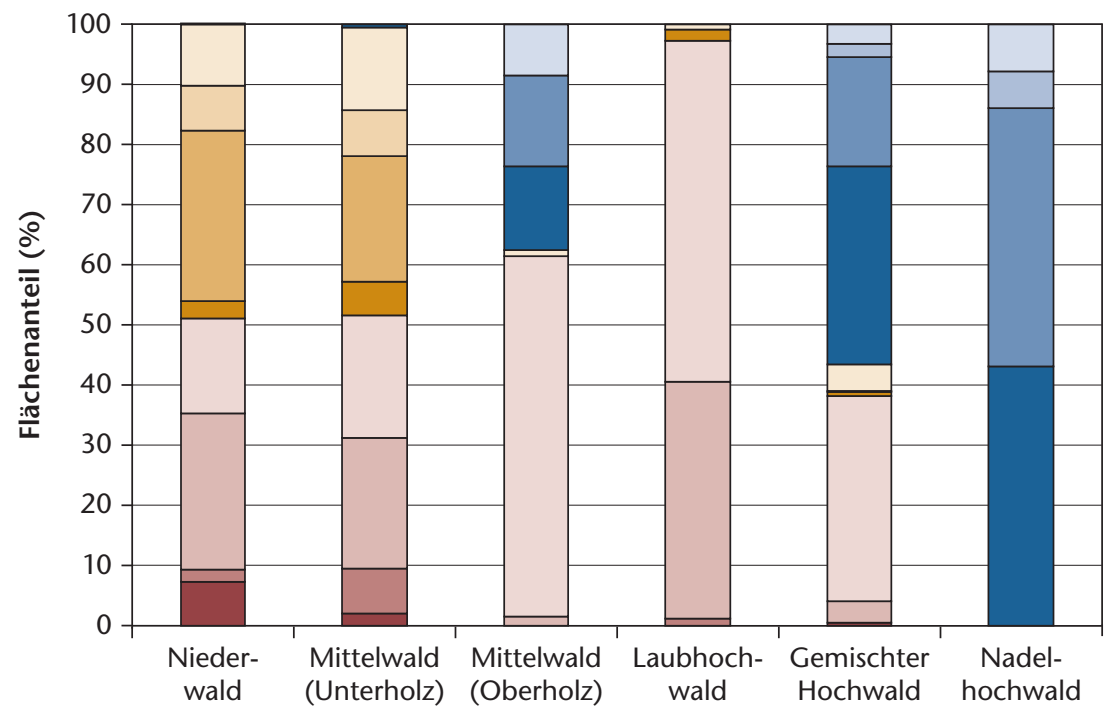

$\square$ Ahorn $\square$ Buche $\square$ Erle $\square$ Linde $\quad \square$ Fichte $\quad \square$ Weisstanne

$\square$ Aspe $\quad$ Eiche $\quad$ Hainbuche $\quad \square$ Laubholz allgemein $\quad \square$ Föhre $\quad \square$ Nadelholz allgemein

Abb 5 Baumartenzusammensetzung pro Betriebsform im Zürcher Unterland im Jahr 1823.

\begin{tabular}{|c|c|c|c|c|c|c|c|c|}
\hline \multirow[t]{2}{*}{ Gemeinde } & \multicolumn{2}{|c|}{ Föhre } & \multicolumn{2}{|c|}{ Fichte } & \multicolumn{2}{|c|}{ Eiche } & \multicolumn{2}{|c|}{ Buche } \\
\hline & $\begin{array}{c}1823 \\
(\%)\end{array}$ & $\begin{array}{c}\text { Heute } \\
(\%)\end{array}$ & $\begin{array}{c}1823 \\
(\%)\end{array}$ & $\begin{array}{c}\text { Heute } \\
(\%)\end{array}$ & $\begin{array}{c}1823 \\
(\%)\end{array}$ & $\begin{array}{c}\text { Heute } \\
(\%)\end{array}$ & $\begin{array}{c}1823 \\
(\%)\end{array}$ & $\begin{array}{c}\text { Heute } \\
(\%)\end{array}$ \\
\hline Bachenbülach & 4.9 & 11.8 & 0.0 & 13.2 & 56.5 & 20.5 & 4.1 & 25.7 \\
\hline Bachs & 42.6 & 16.6 & 21.3 & 27.6 & 18.0 & 9.4 & 9.5 & 27.5 \\
\hline Boppelsen & 9.6 & 4.8 & 4.0 & 18.6 & 12.5 & 9.9 & 26.7 & 36.6 \\
\hline Buchs & 10.2 & 2.9 & 9.2 & 28.8 & 29.6 & 16.0 & 4.6 & 22.0 \\
\hline Dänikon & 0.0 & 0.0 & 23.0 & 33.0 & 27.0 & 0.0 & 10.5 & 33.5 \\
\hline Dielsdorf & 13.6 & 4.6 & 17.6 & 34.6 & 22.5 & 4.9 & 17.3 & 27.4 \\
\hline Dietlikon & 26.9 & 7.9 & 37.0 & 38.4 & 21.4 & 8.6 & 0.0 & 8.7 \\
\hline Hochfelden & 14.0 & 16.1 & 7.2 & 20.8 & 38.5 & 27.7 & 17.1 & 20.6 \\
\hline Höri & 20.0 & 17.9 & 0.0 & 5.5 & 40.0 & 12.5 & 25.0 & 58.8 \\
\hline Kloten & 3.7 & 2.4 & 2.9 & 27.9 & 52.0 & 15.8 & 0.8 & 17.5 \\
\hline Lufingen & 34.0 & 14.5 & 19.8 & 39.1 & 22.6 & 6.9 & 9.0 & 18.4 \\
\hline Niederhasli & 0.0 & 0.0 & 0.0 & 14.1 & 44.4 & 37.7 & 0.0 & 6.2 \\
\hline Niederweningen & 5.4 & 1.6 & 5.6 & 32.6 & 35.4 & 13.1 & 16.2 & 24.8 \\
\hline Nürensdorf & 2.4 & 2.4 & 0.0 & 48.6 & 15.0 & 2.6 & 16.8 & 24.2 \\
\hline Oberembrach & 40.2 & 11.2 & 4.2 & 29.0 & 24.9 & 5.1 & 17.3 & 29.6 \\
\hline Oberengstringen & 10.1 & 0.0 & 19.9 & 18.7 & 38.7 & 11.2 & 10.1 & 13.9 \\
\hline Oberglatt & 6.8 & 0.0 & 35.6 & 14.1 & 57.7 & 29.1 & 0.0 & 13.1 \\
\hline Oberweningen & 12.9 & 6.0 & 0.0 & 34.5 & 47.8 & 12.0 & 12.8 & 26.9 \\
\hline Opfikon & 35.9 & 3.6 & 7.2 & 28.8 & 34.9 & 14.0 & 6.4 & 10.8 \\
\hline Otelfingen & 11.4 & 3.1 & 15.8 & 17.9 & 15.9 & 7.5 & 21.8 & 44.5 \\
\hline Regensberg & 12.8 & 1.3 & 17.0 & 42.5 & 10.4 & 4.7 & 20.3 & 23.8 \\
\hline Regensdorf & 0.0 & 1.9 & 25.9 & 35.7 & 27.4 & 3.7 & 15.0 & 17.7 \\
\hline Rümlang & 0.1 & 4.5 & 37.6 & 34.2 & 29.4 & 23.8 & 10.7 & 15.9 \\
\hline Schleinikon & 2.1 & 2.2 & 4.0 & 39.4 & 37.4 & 11.0 & 5.8 & 29.9 \\
\hline Schöfflisdorf & 9.5 & 7.0 & 0.0 & 41.8 & 39.9 & 9.8 & 15.8 & 23.0 \\
\hline Stadel & 32.6 & 28.2 & 4.4 & 16.8 & 37.1 & 17.4 & 16.5 & 20.5 \\
\hline Steinmaur & 28.9 & 6.1 & 10.1 & 32.2 & 22.4 & 9.0 & 0.0 & 18.2 \\
\hline Unterengstringen & 7.6 & 9.8 & 23.3 & 38.4 & 35.9 & 7.7 & 10.3 & 20.2 \\
\hline Wallisellen & 24.0 & 15.5 & 33.8 & 39.4 & 19.7 & 2.3 & 1.4 & 11.2 \\
\hline Weiningen & 11.4 & 6.8 & 30.3 & 14.8 & 27.1 & 4.8 & 15.9 & 43.0 \\
\hline Winkel & 46.0 & 11.3 & 33.2 & 29.7 & 9.6 & 10.5 & 5.6 & 27.0 \\
\hline
\end{tabular}

Tab 2 Flächenanteile der wichtigsten Baumarten im Jahr 1823 und Grundflächenanteile aus der aktuellen Regionalwaldinventur (Quelle: Amt für Landschaft und Natur, Abteilung Wald) für 31 Gemeinden im Zürcher Unterland. geklumpte Verteilung aufweisen, ist die Fichte in fast allen Gemeinden anzutreffen. Das Vorkommen der Buche ist regional sehr verschieden. Während einige Gemeinden südlich der Lägern ziemlich hohe Buchenanteile zeigen, kommt sie im zentralen und östlichen Unterland selten oder gar nicht vor. Insgesamt weist die Buche einen Anteil von 12\% auf.

Niederwälder und das Unterholz von Mittelwäldern zeigen eine sehr ähnliche Baumartenzusammensetzung (Abbildung 5). Die wichtigsten Baumarten sind Buche, Eiche und Hainbuche. Ahorn, Aspe, Erle und Linde werden ebenfalls oft erwähnt. Im Oberholz von Mittelwäldern ist die Eiche mit einem Anteil von etwa 60\% die wichtigste Baumart, wobei auch Nadelbäume wie Fichte und Föhre häufig vorkommen. Laubhochwälder sind fast immer aus Eichen und Buchen zusammengesetzt. Beide Baumarten scheinen oft in Reinbeständen vorgekommen zu sein. Gemischte Hochwälder bestehen zu etwas mehr als der Hälfte aus Nadelholz. Der Laubholzanteil wird zum grössten Teil aus Eichen gebildet. In Nadelhochwäldern sind Fichte und Föhre zu etwa gleichen Anteilen vertreten, die Weisstanne spielt hier nur eine untergeordnete Rolle.

\section{Veränderung der Baumartenzusammen- setzung im Zürcher Unterland}

Die Baumartenzusammensetzung im Zürcher Unterland hat sich in den letzten 200 Jahren stark verändert (Tabelle 2). So ergibt der Wilcoxon-Test, dass sich die Anteile von Eiche, Buche, Föhre und Fichte - den vier wichtigsten Baumarten im Zürcher Unterland - zwischen 1823 und heute signifikant unterscheiden ( $\mathrm{P}<0.001$; Abbildung 6). Der Föhrenanteil ist in praktisch allen untersuchten Gemeinden seit 1823 stark gesunken. Nur in einigen wenigen Gemeinden ist er konstant geblieben oder sogar leicht angestiegen. Dies ist vor allem dort der Fall, wo der Föhrenanteil schon 1823 relativ gering war. Stark zugenommen hat seit 1823 der Fichtenanteil. Diese Entwicklung kann mit wenigen Ausnahmen in allen Gemeinden beobachtet werden. Einen stark gegenläufigen Trend zeigen nur Oberglatt und Weiningen. Der Eichenanteil ist seit 1823 in fast allen Gemeinden sehr deutlich zurückgegangen. Umgekehrt ist es bei der Buche, deren Anteil in allen untersuchten Gemeinden stark angestiegen ist.

\section{Diskussion}

Wie die Auswertungen zeigen, hat die Bewirtschaftung einen sehr grossen Einfluss auf die Baumartenzusammensetzung. So unterscheiden sich die verschiedenen Betriebsformen in ihrer Baumartenzusammensetzung erheblich. Sehr ähnlich ist die Baumartenzusammensetzung jedoch in Niederwäldern und im Unterholz von Mittelwäldern. Sie besteht dort 

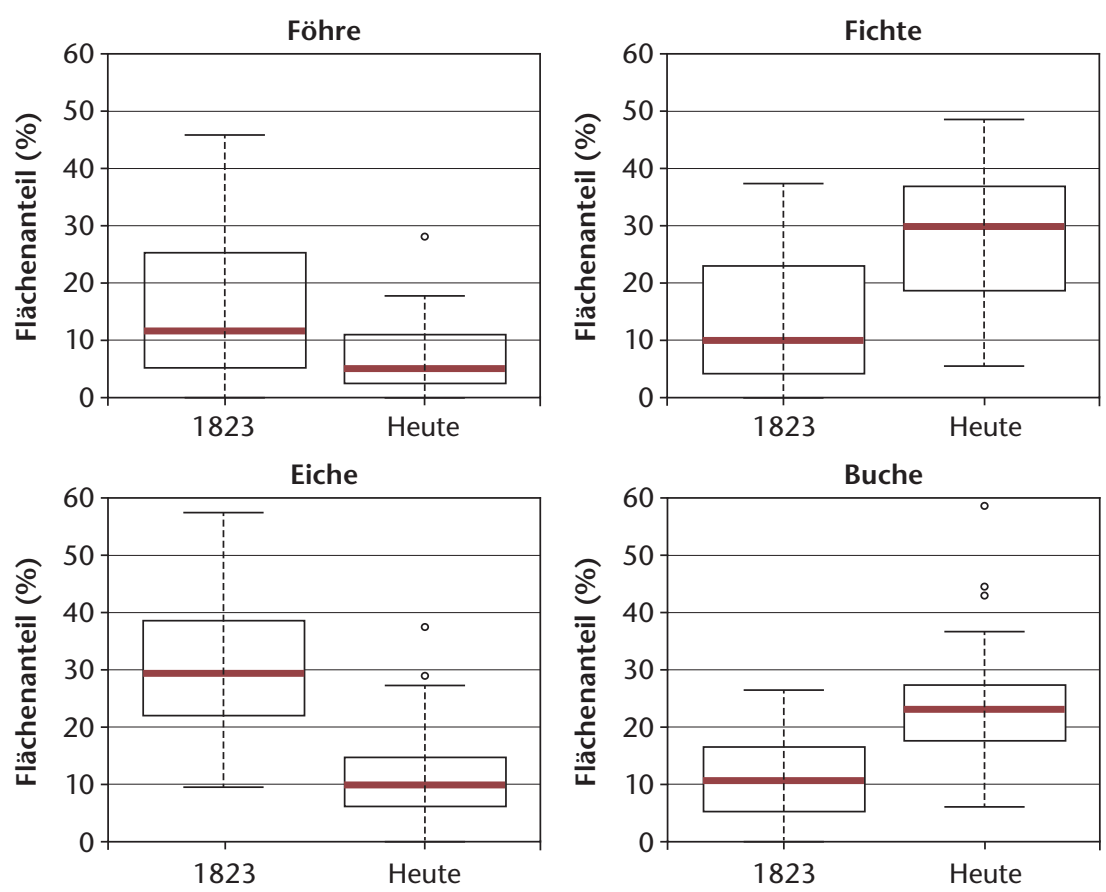

Abb 6 Vergleich der Anteile ausgewählter Baumarten pro Gemeinde im Jahr 1823 (Flächenanteile) und heute (Grundflächenanteile) im Zürcher Unterland ( $n=31)$.

fast ausschliesslich aus Laubholz, weil Nadelbäume keine Stockausschläge bilden können. Dieser Aufbau ist das Resultat einer intensiven Bewirtschaftung mit kurzer Umtriebszeit, bei der die Stockausschläge regelmässig zur Gewinnung von Brennholz abgeschlagen werden. In Niederwäldern und im Unterholz von Mittelwäldern findet sich die grösste Baumartenvielfalt. Dies dürfte darauf zurückzuführen sein, dass durch die intensive Bewirtschaftung sehr viel Licht in die Bestände gelangt und somit auch lichtbedürftige, konkurrenzschwache Pionierbaumarten wie Birken, Pappeln und Weiden aufkommen können.

Stark mit der Mittelwaldbewirtschaftung verbunden ist das Vorkommen der Eiche. Sie bildet in vielen Mittelwäldern den Hauptbestandteil des Oberholzes. Allerdings sind in vielen Gemeinden auch die Fichte und die Föhre stark im Oberholz vertreten. Vermutlich wurden sie wegen ihrer Qualitäten als Bauholz gefördert. Diese Beobachtung machte auch Grossmann (1925) für die erste Hälfte des 20. Jahrhunderts. Die Zusammensetzung des Oberholzes im Mittelwald scheint im 19. Jahrhundert also relativ konstant geblieben zu sein. Die Auswertungen machen auch deutlich, dass die Mittelwälder relativ baumartenreich gewesen sind und keineswegs nur aus Eichen im Ober- und Hainbuchen im Unterholz bestanden.

Die wichtigste Hochwaldform war 1823 der Nadelhochwald. Die Hauptbaumarten sind hier Fichte und Föhre, welche sowohl in Reinbeständen als auch gemischt vorkommen. Die Weisstanne spielt nur eine untergeordnete Rolle. Mit Ausnahme der Lärche werden in den Visitationsberichten keine weiteren Nadelbaumarten genannt. Exoten wie Douglasie und Strobe spielten damals ausserhalb von Pär- ken und Versuchsflächen keine Rolle (Schuler 1976). Viele der reinen Nadelholzbestände sind wahrscheinlich durch Saat oder Pflanzung begründet worden. Die künstliche Verjüngung der Fichte war damals jedoch noch nicht überall verbreitet. Sie begann sich wohl erst ab den 1830er-Jahren grossflächig durchzusetzen (Bürgi \& Schuler 2003). Diese Vermutung wird durch die Tatsache gestützt, dass der Fichtenanteil nach 1823 stark zugenommen hat. Anders scheint es sich bei der Föhre zu verhalten, die 1823 stärker vertreten war als heute. Föhrensaaten dürften daher wohl schon im 18. Jahrhundert eine Rolle gespielt haben. Ausserdem förderte die intensive Nutzung durch Waldweide und Entnahme der Laubstreue die natürliche Verjüngung der Föhre (Grossmann 1925). Im Gegensatz dazu sind Laubhochwälder und gemischte Hochwälder damals vermutlich meist durch natürliche Verjüngung entstanden. Laut Bürgi \& Schuler (2003) machten Laubbäume zu Beginn des 19. Jahrhunderts nur einen kleinen Teil der künstlichen Verjüngung aus.

Der hohe Eichenanteil um 1823 ist mit der damals weit verbreiteten Mittelwaldbewirtschaftung verbunden. Da diese Betriebsform heute kaum mehr vorkommt, hat der Eichenanteil stark abgenommen. Wie Altman et al (2013) mit dendrochronologischen Methoden festgestellt hat, konnten sich die Eichen im Oberholz vor allem nach starken Eingriffen im Unterholz etablieren und aufwachsen. Da solch starke Auflichtungen heute kaum mehr vorgenommen werden, hat die Eiche grosse Schwierigkeiten, sich zu verjüngen. Von dieser Entwicklung profitiert hat dagegen die Buche, die in Mittelwäldern aufgrund ihres geringen Stockausschlagvermögens zurückgedrängt worden war (Schmider et al 1993). Einzig an den steilen Südhängen der Lägern war die Buche schon damals relativ gut vertreten. Vermutlich wurden diese Wälder kaum beweidet, wodurch die Buche vermehrt aufkommen konnte.

Es lässt sich vermuten, dass der Standort einen gewissen Einfluss auf die Wahl der Betriebsform hatte. So finden sich eichenreiche Mittelwälder vor allem in den tiefer gelegenen und niederschlagsärmeren Teilen des Kantons, während Nadelhochwälder in den höher gelegenen Gebieten zu finden sind. Aus Bürgi et al (2010) ist zudem bekannt, dass an den Steilhängen der Lägern Niederwälder vorherrschten. Umfassend lassen sich diese Zusammenhänge aufgrund der Visitationsberichte aber nicht überprüfen, da sich die Bestände aufgrund der ungenauen Ortsangaben nur schwer lokalisieren lassen.

\section{Schlussfolgerungen und Ausblick}

Die intensive Nutzung der Wälder im frühen 19. Jahrhundert führte dazu, dass ihre Zusammensetzung keineswegs einem natürlichen Zustand ent- 
sprach. Wahrscheinlich hatte die Bewirtschaftung bereits 1823 einen grösseren Einfluss auf die Baumartenzusammensetzung als natürliche Faktoren wie Klima, Topografie und Boden. Die Wahl der Betriebsform, welche wiederum die Baumartenzusammensetzung beeinflusst, scheint aber teilweise durch diese Faktoren bestimmt worden zu sein. Während in höher gelegenen und niederschlagsreichen Gebieten die Nadelhochwälder dominierten, fanden sich Mittelwälder vor allem im Norden des Kantons mit geringeren Jahresniederschlägen. Das Aussehen der Wälder um 1823 war somit das Ergebnis einer schon seit mehreren Jahrhunderten andauernden Nutzung und Bewirtschaftung.

Durch die naturnahe Waldbewirtschaftung und die vermehrte Naturverjüngung der letzten Jahrzehnte zeigen die Wälder heute vermutlich ein natürlicheres Aussehen als damals (Bürgi \& Schuler 2003). Allerdings sind die naturbelassenen Wälder im Kanton Zürich oft artenarm und gleichförmig und bestehen auf den meisten Standorten aus praktisch reinen Buchenbeständen. Dies ist sowohl aus Sicht des Natur- und Artenschutzes als auch aus ästhetischen Gründen nicht immer ideal (Schütz 2005). An gewissen Orten kann es daher durchaus sinnvoll sein, traditionelle Betriebsformen zu reaktivieren. So sind Nieder- und Mittelwälder sehr wertvolle Habitate für zahlreiche lichtbedürftige Krautpflanzen und Baumarten (Bartha et al 2008, Määttänen \& Holderegger 2008, Bürgi et al 2010) sowie selten gewordene Schmetterlings- und Käferarten (Fartmann et al 2013, Coch \& Vögeli 2006). Bei der Förderung von Eichen und damit assoziierten Vogelarten wie dem Mittelspecht ist man ebenfalls auf lichte Verhältnisse, wie sie in Mittelwäldern vorkommen, angewiesen (Miranda et al 2006).

Das Wissen über die frühere Bewirtschaftung und Baumartenzusammensetzung ist nicht nur aus rein wissenschaftlicher Sicht interessant. Vielmehr können historische Informationen auch in der Praxis gewinnbringend eingesetzt werden, zum Beispiel als Referenz für die Revitalisierung ehemaliger Nieder- und Mittelwälder im Rahmen von Naturschutzprojekten. Durch die Auswertung historischer Quellen erhält man eine genauere Vorstellung davon, wie die Waldbestände aufgebaut und gepflegt werden sollten. Werden zusätzlich Standortfaktoren wie Klima, Topografie und Boden in die Auswertungen einbezogen, wären vermutlich weitere Erkenntnisse zur Naturnähe der früheren und heutigen Wälder möglich. Ausserdem könnte dadurch der Einfluss der Bewirtschaftung in den verschiedenen Regionen quantifiziert sowie dem Zusammenhang zwischen Standort und Betriebsform nachgegangen werden.

Eingereicht: 26. August 2014, akzeptiert (Review): 6. November 2014

\section{Dank}

Das Amt für Landschaft und Natur des Kantons Zürich hat die Daten aus der Regionalwaldinventur zur Verfügung gestellt. Hier gebührt Hermann Hess ein besonderer Dank für die freundliche Beratung. Ausserdem sei den Mitarbeitern des Staatsarchivs Zürich herzlich für die Bereitstellung der Archivalien und die Beantwortung von Fragen gedankt.

\section{Literatur}

ALTMAN J, HÉDL, R, SZABÓ P, MAZUREK P, RIEDL V ET AL (2013) Tree-rings mirror management legacy: Dramatic response of standard oaks to past coppicing in Central Europe. PLoS ONE 8(2): e55770. doi:10.1371/journal.pone.0055770

AXELSSON AL, ÖSTLUND L (2001) Retrospective gap analysis in a Swedish boreal forest landscape using historical data. For Ecol Manage 147: 109-122.

BARTHA S, MEROLLI A, CAMPETELLA G, CANULLO R (2008) Changes of vascular plant diversity along a chronosequence of beech coppice stands, central Apennines, Italy. Plant Biosyst 142: 572-583.

BÜRGI M (1998) Waldentwicklung im 19. und 20. Jahrhundert. Veränderungen in der Nutzung und Bewirtschaftung des Waldes und seiner Eigenschaften als Habitat am Beispiel der öffentlichen Waldungen im Zürcher Unter- und Weinland. Beih Schweiz Z Forstwes 84. 234 p.

BÜRGI M, RUSSELL EWB, MOTZKIN G (2000) Effects of postsettlement human activities on forest composition in the north-eastern United States: a comparative approach. J Biogeogr 27: 1123-1138.

BÜRGI M, SCHULER A (2003) Driving forces of forest management - an analysis of regeneration practices in the forests of the Swiss Central Plateau during the $19^{\text {th }}$ and $20^{\text {th }}$ century. For Ecol Manage 176: 173-183.

BÜRGI M, STECK C, BERTILLER R (2010) Evaluating a forest conservation plan with historical vegetation data. A transdisciplinary case study from the Swiss lowlands. Gaia 19: 204-212.

COCH T, VÖGELI M (2006) Kronenbewohnende Bockkäfer als Entscheidungshilfen zur Revitalisierung ehemaliger Mittelwälder. Schweiz Z Forstwes 157: 318-324. doi: 10.3188/szf.2006.0318

COGBILL CV, BURK J, MOTZKIN G (2002) The forests of presettlement New England, USA: spatial and compositional patterns based on town proprietor surveys. J Biogeogr 29: 1279-1304.

DUPUIS S, ARSENEAULT D, SIROIS L (2011) Change from pre-settlement to present-day forest composition reconstructed from early land survey records in eastern Québec, Canada. J Veg SCi 22: 564-575.

FARTMANN T, MÜLLER C, PONIATOWSKI D (2013) Effects of coppicing on butterfly communities of woodlands. Biol conserv 159: 396-404.

GIMMI U, WOHLGEMUTH T, RIGLING A, HOFFMANN CW, BÜRGI M (2010) Land-use and climate change effects in forest compositional trajectories in a dry Central-Alpine valley. Annals For Sci 67: 701.

GROSSMANN H (1925) Das Vorkommen der Waldföhre (Pinus silvestris L.) im Norden des Kantons Zürich. Schweiz Z Forstwes 76: 113-131.

MÄÄTTÄNEN K, HOLDEREGGER R (2008) Populationsgeschichte und Managementprinzipien in Schweizer Wildbirnenvorkommen. Schweiz Z Forstwes 159: 8-16. doi: 10.3188/szf.2008.0008

MIRANDA B, SCHIEGG K, BÜHLMANN J, PASINELLI G (2006) Eichenförderungsmassnahmen im Niderholz (Kanton Zürich): Auswirkungen auf Bestand und Bruthöhlenstandorte von Mittel- und Buntspecht. Schweiz Z Forstwes 157: 333-338. 
NOBLE IR, DIRZO R (1997) Forests as human-dominated ecosystems. Science 277: 522-525.

RÜTHER C (2010) The history of vegetation dynamics and forest use in the Leopolds-Hochwald, a forest area in the upper range of the Western Bavarian Forest (Vorderer Bayerischer Wald) - a contribution to the importance of forest-historical, archival documents. Allg Forst- Jagdztg 181: 89-104.

SCHMIDER P, KÜPER M, TSCHANDER B, KÄSER B (1993) Die Waldstandorte im Kanton Zürich. Zürich: VDF. 287 p.

SCHULER A (1976) Zur Geschichte der fremdländischen Baumarten im Schweizer Wald. Schweiz Z Forstwes 127: 205-226.

SCHÜTZ JP (2005) Intensität der Waldpflege und Baumartendiversität im Wald - oder: Naturautomation contra Entmischung. Schweiz Z Forstwes 156: 200-206. doi: 10.3188/ szf.2005.0200

SHOLES ODV, HAWKINS DL (2007) Composition and temporal change of the forest in 18th century Shrewsbury, Massachusetts. J Torrey Bot Soc 134: 74-79.

\section{Les forêts zurichoises en 1823 - formes d'exploitation et essences forestières}

Cette étude reconstruit au moyen des inventaires forestiers de 1823 l'exploitation et la composition des forêts publiques du canton de Zurich à l'aube du XIXe siècle. A cette fin, la répartition des formes d'exploitation est analysée dans l'ensemble du canton, tout comme la composition en essences forestières dans 31 communes de I'Unterland zurichois. En complément, une comparaison avec la composition actuelle des forêts dans ces communes est effectuée. L'évaluation des données démontre que le taillis sous futaie était la forme d'exploitation la plus importante au début du XIXe siècle. La composition des forêts varie fortement en fonction de leur forme d'exploitation, et les taillis ainsi que les taillis sous futaie affichent la diversité en espèces d'arbre la plus importante. Les résultats suggèrent qu'il n'existe pas de relation claire entre la composition d'essences forestières et des facteurs naturels tels que le climat, la topographie ou le sol. Cette constatation permet de conclure qu'au début du XIXe siècle, la gestion avait déjà une plus grande influence sur la composition des essences que les conditions naturelles. Les forêts de l'Unterland zurichois ont fortement évolué au cours des 200 dernières années. Les forêts actuelles devraient être plus proches de l'état naturel que celles de 1823, ce qui n'implique cependant pas une plus grande diversité en espèces. La connaissance des essences forestières qui composaient les forêts par le passé peut ainsi s'avérer utile dans le cadre de revitalisations d'anciens taillis ou taillis sous futaie, qui offrent de précieux habitats à des espèces animales et végétales menacées.
SJÖLUND MJ, JUMP AS (2013) The benefits and hazards of exploiting vegetative regeneration for forest conservation management in a warming world. Forestry 86: 503-513.

TERRAIL R, ARSENEAULT D, FORTIN MJ, DUPUIS S, BOUCHER Y (2014) An early forest inventory indicates high accuracy of forest composition data in pre-settlement land survey records. J Veg Sci 25: 691-702.

VADEBONCOEUR MA, HAMBURG SP, COGBILL CV, SUGIMURA WY (2012) A comparison of presettlement and modern forest composition along an elevation gradient in central New Hampshire. Can J For Res 42: 190-202.

WEISZ L, GROSSMANN H, KREBS E, RITZLER K, OLDANI C (1983) 650 Jahre Zürcherische Forstgeschichte. Band II. Zürich: Berichthaus AG. 481 p.

ZINGG A (2001) Waldbewirtschaftung: Umgang mit der Vergangenheit - für die Zukunft. Schweiz Z Forstwes 152: 177-182. doi: 10.3188/szf.2001.0177

\section{The forests in the Canton of Zurich around 1823 - forest types and tree species}

Based on early forest management plans from 1823, this paper analyses forest management practices and tree species composition in the public forests of the Canton of Zurich in the early $19^{\text {th }}$ century. Forest type distribution across the canton is reconstructed and detailed tree species composition is shown for 31 communities in the north-western part of the canton. For these communities, we compared the historical tree species composition with modern data from a regional forest inventory. The results show that coppice with standards was the most important forest type in the early $19^{\text {th }}$ century. Tree species composition largely depends on the forest types. The highest diversity in tree species can be found in coppice and coppice-with-standards forests. The analyses reveal no clear relationship between tree species composition and environmental factors such as climate, topography and soil. This leads to the conclusion that in the early $19^{\text {th }}$ century already management had a stronger influence on tree composition than natural conditions. During the last 200 years, tree species composition in the Canton of Zurich changed profoundly. Whereas today's forests are more natural in terms of species composition, they are less diverse in species. Knowing the tree species composition in the past can thus be helpful in conservation projects, e.g. in establishing coppice and coppice-with-standards forests in order to promote rare light-demanding plant and animal species. 